\title{
Peripartum Antibiotics Exposure and the Risk of Autoimmune and Autism Disorders in the Offspring
}

\author{
Mohammad H. Murad' Joseph A. Murray ${ }^{5}$ Imad Absah² \\ 1Evidence-Based Practice Research Program, Mayo Clinic, Rochester, \\ Minnesota, United States \\ 2Division of Pediatric Gastroenterology and Hepatology, Mayo \\ Clinic, Rochester, Minnesota, United States \\ ${ }^{3}$ Division of Biomedical Statistics and Informatics, Department \\ of Health Sciences Research, Mayo Clinic, Rochester, Minnesota, \\ United States \\ ${ }^{4}$ Microbiome Program, Center for Individualized Medicine, Division \\ of Obstetrics and Gynecology, Department of Surgery, Mayo \\ Clinic, Rochester, Minnesota, United States \\ ${ }^{5}$ Division of Gastroenterology and Hepatology, Mayo Clinic, \\ Rochester, Minnesota, United States
}

Jehad Almasri $\quad$ Ahmed Barazi ${ }^{2} \quad$ Katherine S. King ${ }^{3} \quad$ Marina R. S. Walther-Antonio ${ }^{4}$ Zhen Wang ${ }^{1}$

Address for correspondence Imad Absah, MD, Division of Pediatric Gastroenterology and Hepatology, Mayo Clinic, Rochester, MN, United States (e-mail: Absah.Imad@mayo.edu).

Avicenna J. Med. 2021;3:118-125.

\author{
Abstract \\ Keywords \\ - antibiotics exposure in \\ pregnancy \\ - autism \\ - autoimmune disease \\ - microbiome
}

Background As the use of antibiotics during the peripartum period increases, the incidence of autoimmune disorders and autism spectrum disorders (ASDs) is also increasing. In this study, we aim to assess if antibiotic exposure during the peripartum period affects the incidence of autoimmune diseases and ASD in the offspring.

Methods We identified children ( $<18$ years of age) born in Olmsted County from January 1, 2003 through December 31, 2012. Offspring with celiac disease (CD), inflammatory bowel disease (IBD), or ASD diagnoses were matched to two controls on birth date, index date, mother's age at delivery, and sex. Data from the mother's medical records were retrieved to determine peripartum antibiotics use.

Results A total of 242 cases and 484 matched controls were included in this study. Median age at the last follow-up was 11.3 years (range: $0.5-14.9$ ), $73 \%$ were males in both groups. Odds of CD diagnosis was not statistically different between vaginal delivery with antibiotics compared with vaginal delivery with no antibiotics (odds ratio [OR] $=0.76,95 \%$ confidence interval $[\mathrm{Cl}]: 0.32-1.85)$, similarly in IBD $(\mathrm{OR}=2.41,95 \% \mathrm{Cl}$ : $0.53-10.98)$ and ASD (OR $=1.00,95 \% \mathrm{Cl}: 0.55-1.79)$. Preeclampsia or eclampsia was associated with offspring $C D(O R=3.20,95 \% \mathrm{Cl}$ : 1.05-9.78). Smoking history and diabetes mellitus were associated with offspring ASD (OR $=1.84,95 \% \mathrm{Cl}: 1.22-2.77$ and $\mathrm{OR}=2.01,95 \% \mathrm{Cl}: 1.03-3.91$, respectively).

Conclusion In this cohort, we found no statistically significant association between peripartum antibiotics exposure and the development of CD, IBD, or ASD. published online August 12, 2021
DOI https://doi.org/

$10.1055 / \mathrm{s}-0041-1732485$

ISSN 2231-0770
C 2021. Syrian American Medical Society.

This is an open access article published by Thieme under the terms of the Creative Commons Attribution-NonDerivative-NonCommercial-License, permitting copying and reproduction so long as the original work is given appropriate credit. Contents may not be used for commercial purposes, or adapted, remixed, transformed or built upon. (https://creativecommons.org/licenses/by-nc-nd/4.0/).

Thieme Medical and Scientific Publishers Pvt. Ltd. A-12, 2nd Floor, Sector 2, Noida-201301 UP, India 


\section{Introduction}

The use of antibiotics during pregnancy has increased over the last two decades. The risk of devastating complications of group B streptococcus (GBS) perinatal infection like sepsis and meningitis has resulted in the Centers for Disease Control and Prevention (CDC) and American College of Obstetricians and Gynecologists (ACOG) recommending the use of prophylaxis antibiotics in GBS+ mothers. ${ }^{1}$ The direct implication of antibiotic exposure on the offspring of those mothers is not well studied. Similarly, the risk of autoimmune diseases in offspring is not clear. Incidence of autoimmune disease is increasing worldwide, approximately 1 of every 31 Americans is affected by at least one autoimmune disease. $^{2}$

A recent study from Olmsted County reported a 2.7-fold increase in the incidence of celiac disease (CD) in children. ${ }^{2}$ This increase is not limited to CD but extends to other autoimmune and allergy-related disorders such as asthma, eczema, and inflammatory bowel disease (IBD). ${ }^{3}$ Additionally, emerging data are showing an increase in autism spectrum disorders (ASDs) prevalence since 2000. ${ }^{4}$ Data set from the CDC suggested a threefold increase in ASD prevalence over the last decade with a current prevalence rate of $18.5 \%(\sim 1$ in 54 children). ${ }^{4}$

The underlying etiology for the observed increment in autoimmune diseases and ASD occurrence is not clear. One hypothesis is that factors which can alter the microbiome during peripartum (last week of gestation and/or during delivery) period, such as antibiotics exposure, infections, and the environment, may play a role. Antibiotics can lead to a perturbation in the neonatal gut microbiome, negatively affecting the development of the immune system. This perturbation may result with increased risk of autoimmune diseases and ASD. ${ }^{5}$ Two previous reports concluded that cesarean section (C-section) as a mode of delivery is associated with increased risk of $\mathrm{CD} .{ }^{6}$ On the contrary, two more recent reports found no significant impact of delivery mode on the risk of $C D .^{7,8}$ No reports have addressed the peripartum antibiotics exposure effect on the risk of developing the autoimmune diseases. An interesting association has been reported between gastrointestinal problems in children and ASD with a possible role for gut microbiota in regulating behaviors and ASD subsequently. ${ }^{9}$

Before 2010, there was inconsistent use of antibiotics before or after the C-section delivery. In 2010, the ACOG guidelines recommended administering antibiotics before all C-section deliveries. There is no clear evidence that these recommendations lead to changes in the overall increase in the incidence of autoimmune diseases or ASD. ${ }^{1}$

The current study aims to assess the association of peripartum antibiotics use with CD, IBD, and ASD.

\section{Materials and Methods}

\section{Study Design}

This is a retrospective case-control study. The Mayo Clinic and Olmsted Medical Center institutional review boards approved the study. Rochester Epidemiology Project (REP) is the source of our data. It involves using three medical records from Mayo Clinic and its affiliated hospitals, Olmsted Medical Center and its affiliated hospital, and the Rochester Family Medicine Clinic.

In our study, we assumed that all C-section cases received peripartum antibiotics and case confirmation was performed in $10 \%$. We compared them to vaginal delivery with antibiotics exposure (Vw) and vaginal delivery without antibiotics exposure (Vw/o) groups. Also, knowing that females have a higher incidence of several autoimmune diseases and males have a higher incidence of ASD (more than 4 to 1), 4,10 we investigated the interaction between sex and the peripartum use of antibiotics on the risk of autoimmune diseases and ASD.

We abstracted information about the peripartum and the delivery from the mother's record including antibiotic usage, steroids administration, and complications or stressful events (i.e., premature rupture of membranes, uterine rupture, placenta excessive bleeding, vasa previa, preeclampsia, and eclampsia), as well as smoking status. We collected information for both the cases and their matched controls (two controls per case) from the children's records including family history of autoimmune diseases, and preterm status ( $<37$ weeks). Study data were collected and managed using REDCap (Research Electronic Data Capture) electronic data capture tools hosted at Mayo Clinic. ${ }^{11}$

\section{Participants}

Olmsted County residents born between January 1, 2003 and December 31, 2012, who had a research authorization to use their medical records were included. Potential cases of $C D$, IBD, or ASD were identified using the International Classification of Diseases (ICD)-9 and ICD-10 codes (see - Appendix [available online only] for the full set of codes). Two physicians (J.A. and A.B.) reviewed the 285 children who had at least one ICD-9/ICD-10 code for CD, IBD, or ASD. Of these children, 248 were verified manually and confirmed to have the diagnoses based on laboratories, images, pathology, and medical documentation from specialists in these conditions. We excluded 6 children, whom their mothers did not give research authorization leaving us with 242 cases. We identified 71 CD cases (142 controls), 13 IBD cases (26 controls), and 163 ASD cases (326 controls). Five children had more than one of these conditions.

\section{Matching Characteristics and Statistical Analyses}

After identifying cases, controls were identified from the remaining births. Each case was matched to two controls, based on the child's birth date, child's date of last follow-up, child's sex, and mother's age at delivery. Any controls where the mother had not given research authorization were replaced with controls that had research authorization. Just like all other cases, those with multiple conditions were matched to two controls. The amount of balance between the cases and controls on matching characteristics was assessed with the standardized difference as this measure is not affected by sample size like a $p$-value. The difference between 
the cases and controls was considered negligible when the standardized difference was less than 0.1.

Univariate conditional logistic regression accounting for the matched pairs was used to assess the association of the $\mathrm{CD}$, IBD, and ASD with the pregnancy and delivery characteristics. Due to the paucity of the IBD cases, we were unable to perform formal comparisons for the other variables. Two-tailed $p$-values less than 0.05 were considered statistically significant. To assess the potential differences of mechanism between the sexes, we tested the interaction of sex and antibiotic exposure in the development of ASD and CD.

\section{Results}

Cases and controls were similar on matching factors, all with standardized differences $<0.1$ ( $=$ Table 1 ). Five patients had both diagnoses of CD and ASD. Demographic characteristics are summarized in -Table 2. There were no cases of vasa previa or uterine rupture in either group. Median age at last follow-up was 11.3 years (range: $0.5-14.9$ ), $73 \%$ were males in both groups. As would be expected, the ASD group had a higher male percentage compared with CD and IBD (85, 49 , and 54\%, respectively). Among the included 726 births, $62 \%$ had $\mathrm{Vw} / 0,13 \%$ had $\mathrm{Vw}$, and $25 \%$ had C-section delivery ( - Fig. 1). Median mothers' age at the time of delivery was 29 years (range: $16-50$ ). At the time of delivery $8 \%$ of the mothers had diabetes mellitus (DM) and 27\% were current or ex-smokers.

\section{Case Comparisons}

In the analyses that follow, we explore whether the risk of CD, IBD, or ASD increases with a variety of exposures. Most of the studied variables are dichotomous (e.g., preterm vs. full-term). The only exposure with multiple categories is $\mathrm{Vw} / \mathrm{o}, \mathrm{Vw}$, and C-section with $\mathrm{Vw} / \mathrm{o}$ being the reference category in this calculation of odds ratios (ORs).

\section{Celiac Disease}

We identified 71 children who were diagnosed with CD. Univariate analysis ( - Table 3 ) showed no significant association of the odds of CD when comparing Vw $(n=31)$ and C-section $(n=54)$ to $\mathrm{Vw} / \mathrm{o}(n=131)(\mathrm{OR}=0.76,95 \%$ confidence interval $[\mathrm{CI}]: 0.32-1.85$ and $\mathrm{OR}=0.94,95 \%$ CI: $0.48-1.82$, respectively). Preeclampsia/eclampsia ( $n=13$ ) was significantly associated with developing CD in the offspring ( $\mathrm{OR}=3.20,95 \% \mathrm{CI}$ : 1.05-9.78). Though data was limited, in this sample there was no significant association of the odds of CD when we compared preterm $(n=21)$ to full-term children (OR $=0.40,95 \% \mathrm{CI}: 0.11-1.48)$. Mothers' history of DM $(n=19)$ and smoking $(n=48)$ were not significantly associated with $\mathrm{CD}(\mathrm{OR}=0.55,95 \% \mathrm{CI}$ : 0.18-1.73 and OR $=0.88,95 \%$ CI: 0.44-1.78, respectively). The interaction between sex and antibiotics in CD was not significant $(p=0.988)$. In CD, the OR for females were 0.94 (95\% CI: 0.39-2.23) and for males the OR was 0.95 (95\% CI: $0.42-2.11)$.

\section{Inflammatory Bowel Disease}

We identified 13 children who were diagnosed with IBD. Among them $\mathrm{Vw}(n=4), \mathrm{Vw} / \mathrm{o}(n=6)$, and C-section $(n=3)$. None of these children were born preterm or were children to diabetic mothers. Twenty-three percent of the mothers were current or ex-smokers. We found no significant association between peripartum antibiotics exposure and IBD $(\mathrm{OR}=2.41,95 \% \mathrm{CI}: 0.53-10.98$ and $\mathrm{OR}=1.74,95 \% \mathrm{CI}$ : $0.33-9.12$, respectively) (- Table 4).

Table 1 Matching characteristics between the identified cases and controls

\begin{tabular}{|c|c|c|c|}
\hline & Cases $(N=242)$ & Controls $(N=484)$ & $\begin{array}{l}\text { Standardized } \\
\text { difference }^{a}\end{array}$ \\
\hline \multicolumn{4}{|l|}{ Child's birth year } \\
\hline Median & 2005 & 2005 & \multirow[t]{2}{*}{0.001} \\
\hline Range & 2003-2011 & 2003-2011 & \\
\hline \multicolumn{4}{|c|}{ Child's age at last follow-up } \\
\hline Mean (SD) & $10.8(2.8)$ & $10.7(2.8)$ & \multirow[t]{3}{*}{0.018} \\
\hline Median (Q1, Q3) & $11.5(8.9,13.0)$ & $11.2(9.0,12.9)$ & \\
\hline Range & $1.6-14.8$ & $0.5-14.9$ & \\
\hline \multicolumn{4}{|c|}{ Mother's age at delivery } \\
\hline Mean (SD) & $30.0(5.9)$ & $29.9(5.9)$ & \multirow[t]{3}{*}{0.001} \\
\hline Median (Q1, Q3) & $29.5(26.3,33.5)$ & $29.3(26.1,33.8)$ & \\
\hline Range & $15.6-50.2$ & $15.6-49.0$ & \\
\hline \multicolumn{4}{|l|}{ Child's sex } \\
\hline Female & $66(27 \%)$ & $132(27 \%)$ & \multirow[t]{2}{*}{0.000} \\
\hline Male & $176(73 \%)$ & $352(73 \%)$ & \\
\hline
\end{tabular}

Abbreviations: Q, interquartile; SD, standard deviation.

${ }^{a}<0.1$ is considered a negligible difference. 


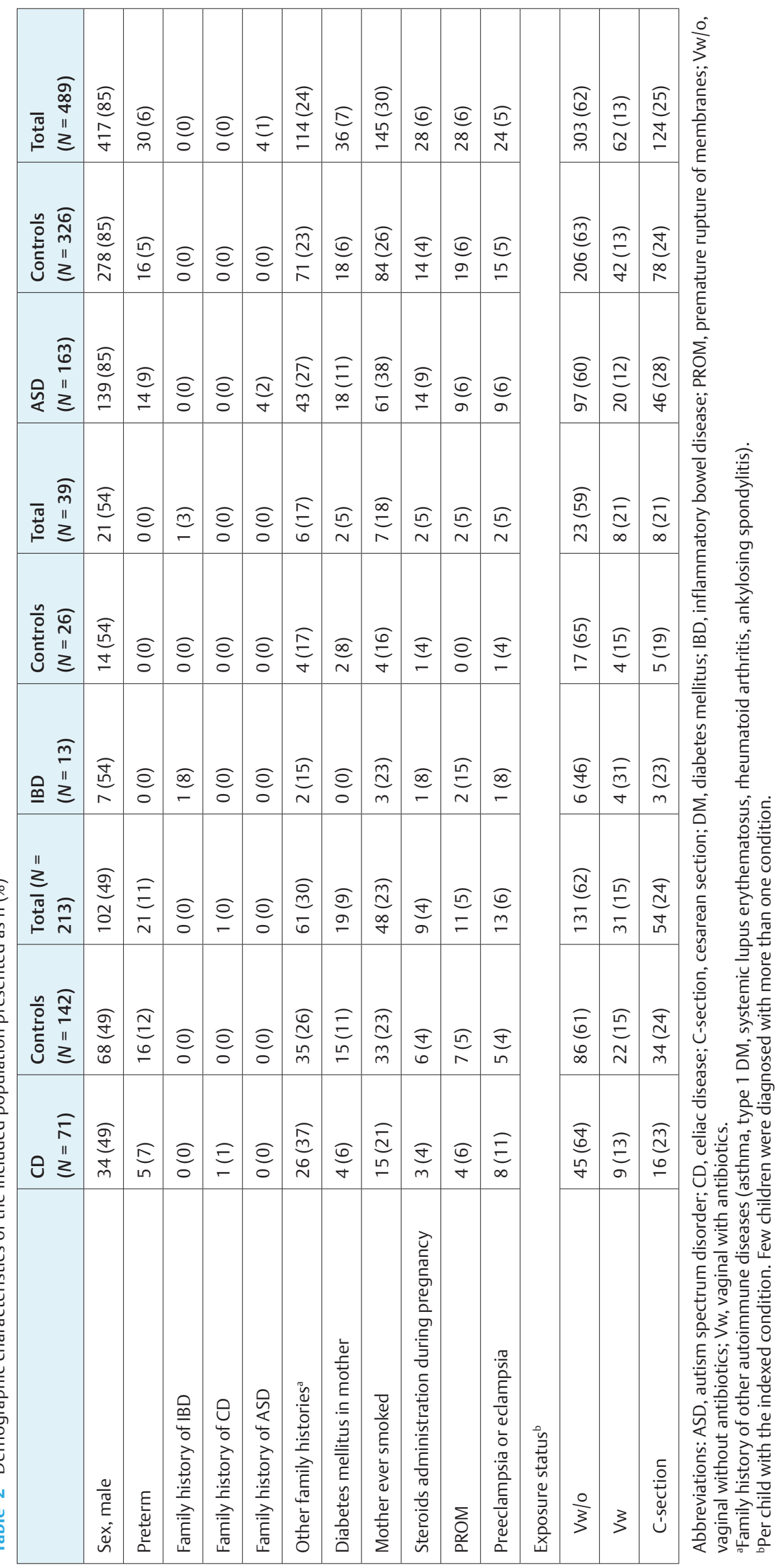



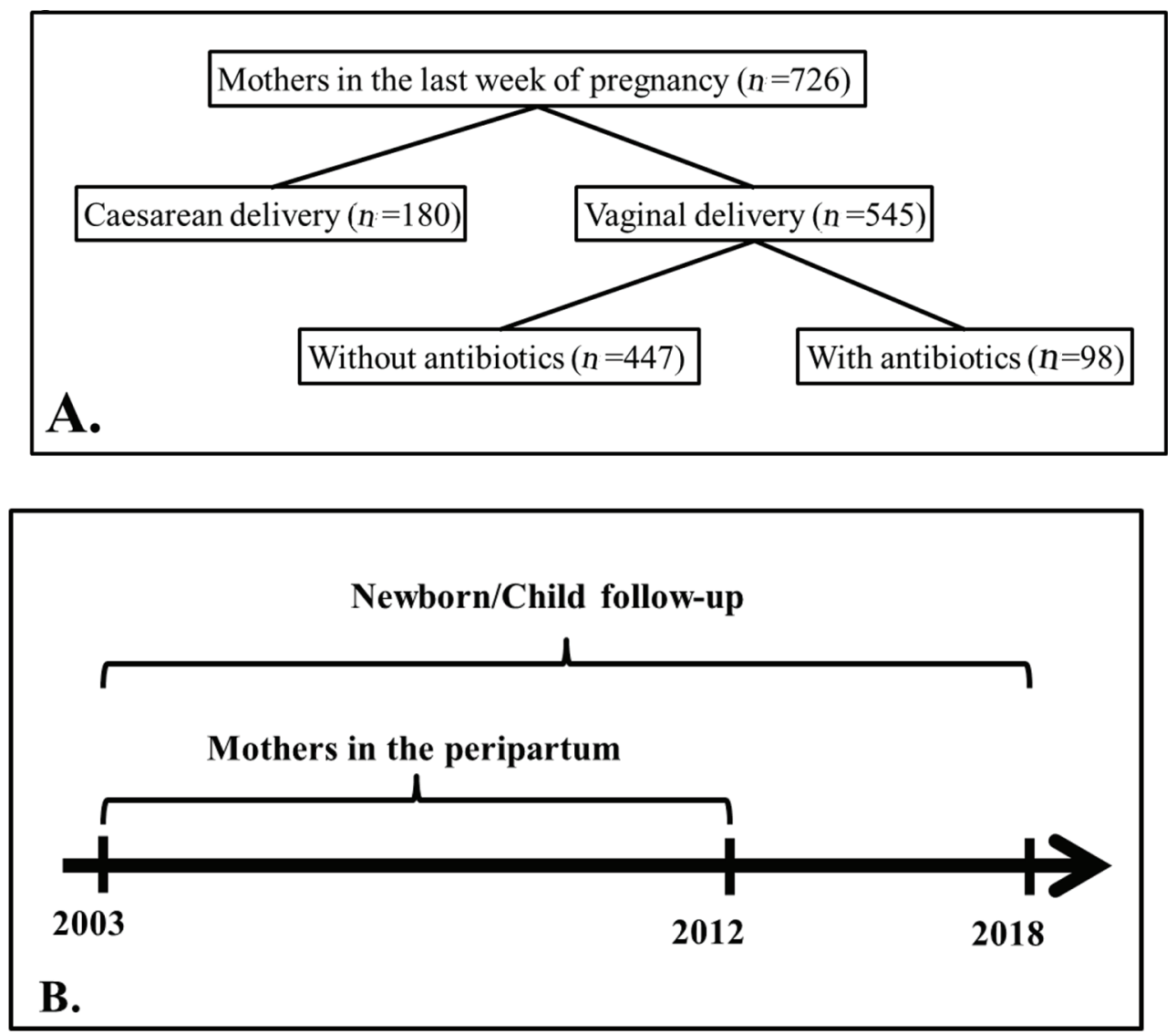

Fig. 1 Study design summary. (A) Flowchart depicts the subgroups of the included mothers per delivery method and per antibiotics exposure for mothers who delivered their babies through the vaginal canal. (The offspring count is matched to the mothers' count per each subgroup.) (B) Overview timeline shows the timeframe of the extracted data of the included mothers who were in the peripartum ranged from 2003 to 2012 and the timeframe of their offspring follow-up ranged from 2003 to 2018 or until they developed one of the conditions of interest.

\section{Autism Spectrum Disorder}

We identified 163 children who were diagnosed with ASD. Neither children in Vw $(n=62)$ nor C-section $(n=124)$ groups had higher odds of ASD than those in the $\mathrm{Vw} / \mathrm{o}$ group $(n=303)(\mathrm{OR}=1.00,95 \% \mathrm{CI}: 0.55-1.79$ and $\mathrm{OR}=1.25,95 \% \mathrm{Cl}$ : $0.81-1.92$, respectively) (- Table 5 ).

Significant associations were detected between ASD diagnosis in offspring and mothers with DM $(n=36)$ and were current or ex-smokers $(n=145)(\mathrm{OR}=2.01,95 \% \mathrm{Cl}$ : $1.03-3.91$ and $\mathrm{OR}=1.84,95 \% \mathrm{CI}: 1.22-2.77$, respectively). No significant association was detected between preterm $(n=30)$ or preeclampsia/eclampsia $(n=24)$ and ASD $(\mathrm{OR}=2.01,95 \% \mathrm{CI}: 0.88-4.60$ and $\mathrm{OR}=1.20,95 \% \mathrm{CI}: 0.53-2.74$, respectively). There was no significant interaction $(p=0.512)$ of sex and antibiotics exposure for the odds of being diagnosed with ASD, the OR for females was 1.59 (95\% CI: 0.62$4.06)$ and 1.12 (95\% CI: 0.73-1.74) for males.

\section{Discussion}

Antibiotics exposure during pregnancy is continuously increasing. ${ }^{1}$ Since 1980 , only $10 \%$ out of 640 medications approved by the U.S. Food and Drug Administration have sufficient evidence of safety and effectiveness during pregnancy. ${ }^{12}$ Several antibiotics have been proven to cause multiple birth defects and are associated with different autoimmune diseases in newborns including asthma and obesity. ${ }^{13}$ It remains important to treat pregnant women with active infections to prevent any possible fetal harm. However, when it comes to treating mild cases with antibiotics more data about the effect of antibiotics on the offspring is needed. The current study adds to the available important data on the association between peripartum antibiotics exposure and the risk of $C D$, IBD, and ASD in the offspring. Identifying the role of antibiotics exposure on developing autoimmune diseases like $C D$ and IBD along with ASD will guide the discussion between the treating provider and the pregnant women to reach an informed and shared decision about the antibiotic use.

In this cohort, we found no significant association between the peripartum antibiotic exposure and developing CD, IBD, and ASD which is consistent with two previous studies. ${ }^{14,15}$ These older studies used data from questionnaires that put their results at risk of recall bias whereas the strength of our data are that it relied on the electronic medical records to confirm the status of the collected data. Additionally, the two aforementioned reports looked at the antibiotics exposure during the whole period of pregnancy. One of the limitations to this approach is of looking at the antibiotics exposure during the whole period of pregnancy. Early antibiotics exposure may not 
Table 3 Univariate conditional logistic odds ratios for CD

\begin{tabular}{|l|l|l|l|}
\hline & $n(\%)$ & OR (95\% CI) & $p$-Value \\
\hline Preterm & $21(11)$ & $0.40(0.11-1.48)$ & 0.17 \\
\hline Diabetes mellitus & $19(9)$ & $0.55(0.18-1.73)$ & 0.31 \\
\hline Mother ever smoked & $48(23)$ & $0.88(0.44-1.78)$ & 0.72 \\
\hline Steroids administration during pregnancy & $9(4)$ & $1.00(0.23-4.35)$ & 1.00 \\
\hline PROM & $11(5)$ & $1.14(0.33-3.90)$ & 0.83 \\
\hline Preeclampsia or eclampsia & $13(6)$ & $3.20(1.05-9.78)$ & 0.04 \\
\hline Exposure status & \multicolumn{3}{|l|}{} \\
\hline Vw/o & $131(62)$ & 1 (reference) & 0.55 \\
\hline Vw & $31(15)$ & $0.76(0.32-1.85)$ & 0.85 \\
\hline C-section & $54(24)$ & $0.94(0.48-1.82)$ & \\
\hline
\end{tabular}

Abbreviations: $\mathrm{CD}$, celiac disease; $\mathrm{Cl}$, confidence interval; $C$-section, cesarean section; $n$, overall total number of events; OR, odds ratio; PROM, premature rupture of membranes; $\mathrm{Vw}$, vaginal with antibiotics; $\mathrm{Vw} / \mathrm{o}$, vaginal without antibiotics.

Table 4 Univariate conditional logistic odds ratios for IBDa

\begin{tabular}{|c|c|c|c|}
\hline & $n(\%)$ & OR $(95 \% \mathrm{Cl})$ & $p$-Value \\
\hline \multicolumn{4}{|c|}{ Exposure status } \\
\hline $\mathrm{Vw} / \mathrm{o}$ & 23 (59) & 1 (reference) & \\
\hline Vw & $8(21)$ & $2.41(0.53-10.98)$ & 0.26 \\
\hline C-section & $8(21)$ & $1.74(0.33-9.12)$ & 0.51 \\
\hline
\end{tabular}

Abbreviations: $\mathrm{Cl}$, confidence interval; C-section, cesarean section; IBD, inflammatory bowel disease; $n$, overall total number of events; OR, odds ratio; $V w$, vaginal with antibiotics; Vw/o, vaginal without antibiotics.

'Other variables not calculated due to the small sample size of cases with IBD.

Table 5 Univariate conditional logistic odds ratios for ASD

\begin{tabular}{|l|l|l|l|}
\hline & $n(\%)$ & OR (95\% Cl) & $p$-Value \\
\hline Preterm & $30(6)$ & $2.01(0.88-4.60)$ & 0.10 \\
\hline Diabetes mellitus & $36(7)$ & $2.01(1.03-3.91)$ & 0.04 \\
\hline Mother ever smoked & $145(30)$ & $1.84(1.22-2.77)$ & 0.003 \\
\hline Steroids administration during pregnancy & $28(6)$ & $2.00(0.95-4.20)$ & 0.07 \\
\hline PROM & $28(6)$ & $0.95(0.43-2.09)$ & 0.89 \\
\hline Preeclampsia or eclampsia & $24(5)$ & $1.20(0.53-2.74)$ & 0.67 \\
\hline Exposure status & & & $1($ reference) \\
\hline Vw/o & $303(62)$ & $1.00(0.55-1.79)$ & 0.99 \\
\hline Vw & $62(13)$ & $124(25)$ & $1.25(0.81-1.92)$ \\
\hline C-section & & 0.31 \\
\hline
\end{tabular}

Abbreviations: ASD, autism spectrum disorder; Cl, confidence interval; C-section, cesarean section; $n$, overall total number of events; OR, odds ratio; PROM, premature rupture of membranes; $\mathrm{Vw}$, vaginal with antibiotic; $\mathrm{Vw} / \mathrm{o}$, vaginal without antibiotics.

result in significant lasting effect on the microbiota, as the usual time for bacteria to recover is 1.5 to 6 months which might happen even before the delivery time. ${ }^{16}$ In our study, we looked at the exposure during the peripartum period to be able to determine the direct effect of antibiotics on the microbiota $^{17}$ and subsequently on the offspring. To our knowledge, this is the first study that investigates the peripartum exposure and risk of $\mathrm{CD}$, IBD, and ASD development in offspring.

In our study, we found no significant association between C-section delivery and $\mathrm{CD}$ which is consistent with previous reports. ${ }^{6,18}$ This suggests that newborn exposure to the maternal vaginal flora may play a role in developing the immune system of those children. In a population-based case-control 
study, an association was found between elective (not emergency) C-section delivery and $\mathrm{CD} .{ }^{19}$ This finding suggest that newborn bacterial flora may affect the development of $C D$. On the other hand, two more recent reports concluded that there is no significant impact of the mode of delivery on the risk of developing $C D{ }^{7.8}$ Our sample on IBD was small but it showed no significant association between IBD and antibiotics exposure or mode of delivery as well. This is consistent with a previous review published in 2014. ${ }^{20}$ Our data showed no association between C-section and ASD, while previous systematic review published in 2015 and pooled data from 13 studies showed a moderate positive association between $\mathrm{C}$-section and ASD development $(\mathrm{OR}=1.23 ; 95 \% \mathrm{CI}$ : $1.07-1.40) .{ }^{21}$ Among those studies, only four studies used United States-based populations and the most recent study published in $2009^{22}$ has a smaller number of cases compared with our study which may have affected the conclusion.

Recently, it has been suggested that there is a relative delay in the colonization of the beneficial intestinal bacteria among preterm infants. ${ }^{23}$ Based on these findings a population-based case-control study was conducted in 2012 and investigated the risk of $\mathrm{CD}$ in preterm infants. The results showed that individuals with preterm birth were less likely to develop CD (OR $=0.87 ; 95 \% \mathrm{CI}: 0.79-0.95) \cdot{ }^{19}$ Our study showed similar findings with OR of 0.40 but was not statistically significant (95\% CI: 0.11-1.48); however, our data are limited by the small number of preterm children $(n=21)$ and the missing data.

In our cohort, we found a positive association between preeclampsia/eclampsia and $\mathrm{CD}$ in the offspring $(\mathrm{OR}=$ 3.20, 95\% CI: 1.05-9.78). A recent population-based cohort study found no significant association between severe preeclampsia/eclampsia and $C D$ but their cohort included only 5 children with $C D$ which may have affected the results. The pathophysiology for such an association is not clear, but it is thought that preeclampsia/eclampsia can compromise the blood flow between the placenta and fetus, which may reduce the fetal gut perfusion, which may result in long-term gastrointestinal morbidities. ${ }^{24}$

In our study, we found significant association between maternal smoking status (current or ex-smoker) and the development of ASD in offspring (OR $=1.84$; $95 \% \mathrm{CI}$ : 1.22 2.77). These results are inconsistent with the findings from recent reports that showed no significant association between smoking and ASD. ${ }^{25,26}$ Given this inconsistency, we separated the results for current and ex-smokers and found significant association only between ex-smoker mothers and ASD (total $n=80$ [17\%], OR $=2.25,95 \% \mathrm{CI}: 1.31-3.85$ ).

\section{Strengths and Limitations}

The current study was established to identify if antibiotic exposure during peripartum is associated with the diseases of interest in their offspring. No other reports addressed the prenatal and intrapartum antibiotics exposure effect on developing $C D$, IBD, and ASD.

This study took advantage of the population-based medical data available through the REP resources with the ability to link mothers to their children. This linked medical records system specifically between mothers and their children allowed us access to accurate and detailed clinical and laboratory data over many years, which are not typically available in other databases or research settings. This resource also allowed us to efficiently examine long-term outcomes retrospectively in a well-defined population in a relatively short period, while a prospective study would require years to collect complete data and will be very expensive.

The primary limitation of this study is its retrospective nature. This study relied on the complete and accurate recording of pertinent information in the electronic medical record. Our data provided preliminary information to support the feasibility and importance of studies to identify the causal links between the microbiome and childhood diseases and conditions. Another limitation of our study relates to the average onset of the studied conditions, which varies from younger than 3 years for $\mathrm{ASD}^{27}$ to around 9 years old to $\mathrm{CD}^{2}$ and IBD. ${ }^{28}$ This could have resulted in missing data of children with less than 5 years' follow-up, especially for $C D$ and IBD conditions. Our sample size was relatively small which reduces our power to detect differences. However, the estimates were small in magnitude and were sometimes in the opposite direction of our expectations although still small. Additionally, the CIs were relatively wide and do not exclude an important association. Therefore, the estimates are imprecise and we cannot be certain in ruling out these associations.

\section{Conclusion}

In this cohort, we found no association between antibiotics exposure during the peripartum period and developing $C D$, IBD, or ASD.

\section{References}

1 Ledger WJ, Blaser MJ. Are we using too many antibiotics during pregnancy. ? BJOG 2013;120(12):1450-1452

2 Almallouhi E, King KS, Patel B, et al. Increasing incidence and altered presentation in a population-based study of pediatric celiac disease in North America. J Pediatr Gastroenterol Nutr 2017;65(4):432-437

3 Asher MI, Montefort S, Björkstén B, et al. ISAAC Phase Three Study Group. Worldwide time trends in the prevalence of symptoms of asthma, allergic rhinoconjunctivitis, and eczema in childhood: ISAAC Phases One and Three repeat multicountry cross-sectional surveys. Lancet 2006;368(9537):733-743

4 (CDC) CfDCaP. Data \& Statistics on Autism Spectrum Disorder. Published 2020. Available at: https://www.cdc. gov/ncbddd/autism/data.html. Updated March 25, 2020. Accessed May 25, 2020

5 Tormo-Badia N, Håkansson Å, Vasudevan K, Molin G, Ahrné S, Cilio CM. Antibiotic treatment of pregnant non-obese diabetic mice leads to altered gut microbiota and intestinal immunological changes in the offspring. Scand J Immunol 2014;80(4):250-260

6 Decker E, Engelmann G, Findeisen A, et al. Cesarean delivery is associated with celiac disease but not inflammatory bowel disease in children. Pediatrics 2010;125(6):e1433-e1440

7 Lionetti E, Castellaneta S, Francavilla R, Pulvirenti A, Catassi C; SIGENP Working Group of Weaning and CD 
Risk. Mode of delivery and risk of celiac disease: risk of celiac disease and age at gluten introduction cohort study. J Pediatr 2017;184:81-86.e2

8 Koletzko S, Lee HS, Beyerlein A, et al. Caesarean section on the risk of celiac disease in the offspring: the Teddy study. J Pediatr Gastroenterol Nutr 2017

9 Sharon G, Cruz NJ, Kang DW, et al. Human gut microbiota from autism spectrum disorder promote behavioral symptoms in mice. Cell 2019;177(6):1600-1618.e17

10 Cooper GS, Stroehla BC. The epidemiology of autoimmune diseases. Autoimmun Rev 2003;2(3):119-125

11 Harris PA, Taylor R, Thielke R, Payne J, Gonzalez N, Conde JG. Research electronic data capture (REDCap)-a metadata-driven methodology and workflow process for providing translational research informatics support. J Biomed Inform 2009;42(2):377-381

12 Adam MP, Polifka JE, Friedman JM. Evolving knowledge of the teratogenicity of medications in human pregnancy. Am J Med Genet C Semin Med Genet 2011;157C(3):175-182

13 Bai L, Zhao D, Cheng Q et al. Trimester-specific association between antibiotics exposure during pregnancy and childhood asthma/wheeze: the role of confounding. Ann Epidemiol 2018

14 Mårild K, Ludvigsson J, Sanz Y, Ludvigsson JF. Antibiotic exposure in pregnancy and risk of coeliac disease in offspring: a cohort study. BMC Gastroenterol 2014;14(1):75

15 Atladóttir HÓ, Henriksen TB, Schendel DE, Parner ET. Autism after infection, febrile episodes, and antibiotic use during pregnancy: an exploratory study. Pediatrics 2012;130(6):e1447-e1454

16 Palleja A, Mikkelsen KH, Forslund SK, et al. Recovery of gut microbiota of healthy adults following antibiotic exposure. Nat Microbiol 2018;3(11):1255-1265

17 Yassour M, Vatanen T, Siljander H, et al. DIABIMMUNE Study Group. Natural history of the infant gut microbiome and impact of antibiotic treatment on bacterial strain diversity and stability. Sci Transl Med 2016;8(343):343ra81

18 Neu J, Rushing J. Cesarean versus vaginal delivery: long-term infant outcomes and the hygiene hypothesis. Clin Perinatol 2011;38(2):321-331
19 Mårild K, Stephansson O, Montgomery S, Murray JA, Ludvigsson JF. Pregnancy outcome and risk of celiac disease in offspring: a nationwide case-control study. Gastroenterology 2012;142(1):39-45.e3

20 Bruce A, Black M, Bhattacharya S. Mode of delivery and risk of inflammatory bowel disease in the offspring: systematic review and meta-analysis of observational studies. Inflamm Bowel Dis 2014;20(7):1217-1226

21 Curran EA, O'Neill SM, Cryan JF, et al. Research review: birth by caesarean section and development of autism spectrum disorder and attention-deficit/hyperactivity disorder: a systematic review and meta-analysis. J Child Psychol Psychiatry 2015;56(5):500-508

22 Bilder D, Pinborough-Zimmerman J, Miller J, McMahon W. Prenatal, perinatal, and neonatal factors associated with autism spectrum disorders. Pediatrics 2009;123(5):1293-1300

23 Westerbeek EA, van den Berg A, Lafeber HN, Knol J, Fetter WP, van Elburg RM. The intestinal bacterial colonisation in preterm infants: a review of the literature. Clin Nutr 2006;25(3):361-368

24 Leybovitz-Haleluya N, Wainstock T, Sheiner E. Maternal preeclampsia and the risk of pediatric gastrointestinal diseases of the offspring: a population-based cohort study. Pregnancy Hypertens 2019;17:144-147

25 Kalkbrenner AE, Braun JM, Durkin MS, et al. Maternal smoking during pregnancy and the prevalence of autism spectrum disorders, using data from the autism and developmental disabilities monitoring network. Environ Health Perspect 2012;120(7):1042-1048

26 Lee BK, Gardner RM, Dal H, et al. Brief report: maternal smoking during pregnancy and autism spectrum disorders. J Autism Dev Disord 2012;42(9):2000-2005

27 Ozonoff S, Heung K, Byrd R, Hansen R, Hertz-Picciotto I. The onset of autism: patterns of symptom emergence in the first years of life. Autism Res 2008;1(6):320-328

28 Rosen MJ, Dhawan A, Saeed SA. Inflammatory bowel disease in children and adolescents. JAMA Pediatr 2015;169(11):1053-1060 